\title{
Some geometrical and algebraic properties of various types of convex hulls
}

\author{
B. Dacorogna \\ Section de mathématiques, EPFL, 1015 Lausanne, Switzerland
}

January 21, 2004

\begin{abstract}
This article is dedicated to Jean Jacques Moreau for his 80th birthday. He is a master of convex analysis.

When dealing with differential inclusions of the form

$$
D u(x) \in E \text {, a.e. in } \Omega
$$

together with some boundary data $u=\varphi$ on $\partial \Omega$, one is lead to consider several types of convex hulls of sets. It is the aim of the present article to discuss these matters.
\end{abstract}

\section{Introduction}

We start in this introduction with the analytical motivations for studying some extensions of the notion of convex hull of a given set. In the remaining part of the article we will however only discuss geometrical and algebraic aspects of these notions. Therefore the reader only interested on these aspects can completely skip the introduction, since we will not use any of the notions discussed now.

We let $\Omega \subset \mathbb{R}^{n}$ be a bounded open set, $u: \Omega \subset \mathbb{R}^{n} \rightarrow \mathbb{R}^{m}$ and therefore the gradient matrix $D u$ belongs to $\mathbb{R}^{m \times n}$ and finally we let $E \subset \mathbb{R}^{m \times n}$ be a compact set.

We are interested in solving the following Dirichlet problem

$$
\left\{\begin{array}{c}
D u(x) \in E, \text { a.e. } x \in \Omega \\
u(x)=\varphi(x), x \in \partial \Omega
\end{array}\right.
$$

where $\varphi: \bar{\Omega} \rightarrow \mathbb{R}^{m}$ is a given map.

In the scalar case $(n=1$ or $m=1)$ a sufficient condition for solving the problem is

$$
D \varphi(x) \in E \cup \operatorname{int} \operatorname{co} E \text {, a.e. in } \Omega
$$

where int co $E$ stands for the interior of the convex hull of $E$. This fact was observed by several authors, with different proofs and different levels of generality; notably in (1), (3), (6), (7), (9), (13) or (14). It should be noted that this 
sufficient condition is very close from the necessary one, which, when properly formulated, is

$$
D \varphi(x) \in \overline{\mathrm{co}} E \text {, a.e. in } \Omega,
$$

where $\overline{\mathrm{co}} E$ denotes the closure of the convex hull of $E$.

When turning to the vectorial case $(n, m \geq 2)$ the problem becomes considerably harder and conditions (1) and (2) are not anymore appropriate. One needs to introduce several extensions of the notion of convex hull, namely the polyconvex, quasiconvex and rank one convex hulls. We will define these notions precisely in the next section, but let us quote first an existence theorem involving the quasiconvex hull, $\overline{\mathrm{Qco}} E$.

We start with the following definition introduced by Dacorogna-Marcellini in (8) (cf. also (9) and (10)), which is the key condition to get existence of solutions.

Definition 1 (Relaxation property) Let $E \subset \mathbb{R}^{m \times n}$. We say that $\overline{\mathrm{Qco}} E$ has the relaxation property if for every bounded open set $\Omega \subset \mathbb{R}^{n}$, for every affine function $u_{\xi}$ satisfying

$$
D u_{\xi}(x)=\xi \in \operatorname{int} \overline{\mathrm{Qco}} E,
$$

there exists a sequence $\left\{u_{\nu}\right\}$ of piecewise affine functions in $\bar{\Omega}$, so that

$$
\begin{aligned}
& u_{\nu} \in u_{\xi}+W_{0}^{1, \infty}\left(\Omega ; \mathbb{R}^{m}\right), D u_{\nu}(x) \in \operatorname{int} \overline{\mathrm{Qco}} E \text {, a.e. in } \Omega \\
& u_{\nu} \stackrel{*}{\rightarrow} u_{\xi} \text { in } W^{1, \infty}, \int_{\Omega} \operatorname{dist}\left(D u_{\nu}(x) ; E\right) d x \rightarrow 0 \text { as } \nu \rightarrow \infty .
\end{aligned}
$$

The main existence theorem is then

Theorem 2 Let $\Omega \subset \mathbb{R}^{n}$ be open. Let $E \subset \mathbb{R}^{m \times n}$ be such that $E$ is compact. Assume that $\overline{\mathrm{Qco}} E$ has the relaxation property. Let $\varphi$ be piecewise $C^{1}\left(\bar{\Omega} ; \mathbb{R}^{m}\right)$ and verifying

$$
D \varphi(x) \in E \cup \operatorname{int} \overline{\mathrm{Qco}} E \text {, a.e. in } \Omega \text {. }
$$

Then there exists (a dense set of) $u \in \varphi+W_{0}^{1, \infty}\left(\Omega ; \mathbb{R}^{m}\right)$ such that

$$
D u(x) \in E \text {, a.e. in } \Omega \text {. }
$$

Remark 3 The theorem was first proved by Dacorogna-Marcellini in (8) (cf. also Theorem 6.3 in (9)) under an extra further hypothesis. This hypothesis was later removed by Sychev in (22) (see also Müller-Sychev (21)), using the method of convex integration of Gromov as revisited by Müller-Sverak (20), and Kirchheim (17). As stated it has been recently proved by Dacorogna-Pisante (10). 


\section{The different types of convex hulls}

We now discuss the central notions of our article, for more background we refer to Dacorogna-Marcellini (9).

We will discuss the different notions of hulls by dual considerations on functions; we therefore start with the following definitions.

Definition 4 (i) A function $f: \mathbb{R}^{m \times n} \rightarrow \overline{\mathbb{R}}=\mathbb{R} \cup\{+\infty\}$ is said to be polyconvex if

$$
f\left(\sum_{i=1}^{\tau+1} t_{i} \xi_{i}\right) \leq \sum_{i=1}^{\tau+1} t_{i} f\left(\xi_{i}\right)
$$

whenever $t_{i} \geq 0$ and

$$
\sum_{i=1}^{\tau+1} t_{i}=1, T\left(\sum_{i=1}^{\tau+1} t_{i} \xi_{i}\right)=\sum_{i=1}^{\tau+1} t_{i} T\left(\xi_{i}\right)
$$

where for a matrix $\xi \in \mathbb{R}^{m \times n}$ we let

$$
T(\xi)=\left(\xi, a d j_{2} \xi, \ldots, a d j_{m \wedge n} \xi\right)
$$

where $a d j_{s} \xi$ stands for the matrix of all $s \times s$ subdeterminants of the matrix $\xi$, $1 \leq s \leq m \wedge n=\min \{m, n\}$ and where

$$
\tau=\tau(m, n)=\sum_{s=1}^{m \wedge n}\left(\begin{array}{c}
m \\
s
\end{array}\right)\left(\begin{array}{c}
n \\
s
\end{array}\right) \text { and }\left(\begin{array}{c}
m \\
s
\end{array}\right)=\frac{m !}{s !(m-s) !}
$$

(ii) A Borel measurable function $f: \mathbb{R}^{m \times n} \rightarrow \mathbb{R}$ is said to be quasiconvex if

$$
\int_{U} f(\xi+D \varphi(x)) d x \geq f(\xi) \operatorname{meas}(U)
$$

for every bounded domain $U \subset \mathbb{R}^{n}, \xi \in \mathbb{R}^{m \times n}$, and $\varphi \in W_{0}^{1, \infty}\left(U ; \mathbb{R}^{m}\right)$.

(iii) A function $f: \mathbb{R}^{m \times n} \rightarrow \overline{\mathbb{R}}=\mathbb{R} \cup\{+\infty\}$ is said to be rank one convex if

$$
f\left(t \xi_{1}+(1-t) \xi_{2}\right) \leq t f\left(\xi_{1}\right)+(1-t) f\left(\xi_{2}\right)
$$

for every $\xi_{1}, \xi_{2}$ with $\operatorname{rank}\left\{\xi_{1}-\xi_{2}\right\}=1$ and every $t \in[0,1]$.

(iv) The different envelopes of a given function $f$ are defined as

$$
\begin{aligned}
& C f=\sup \{g \leq f: g \text { convex }\}, \\
& P f=\sup \{g \leq f: g \text { polyconvex }\}, \\
& Q f=\sup \{g \leq f: g \text { quasiconvex }\}, \\
& R f=\sup \{g \leq f: g \text { rank one convex }\} .
\end{aligned}
$$

We are now in a position to define the main notions of the article. 
Definition 5 We let, for $E \subset \mathbb{R}^{m \times n}$,

$$
\begin{aligned}
& \overline{\mathcal{F}}_{E}=\left\{f: \mathbb{R}^{m \times n} \rightarrow \overline{\mathbb{R}}=\mathbb{R} \cup\{+\infty\}:\left.f\right|_{E} \leq 0\right\} \\
& \mathcal{F}_{E}=\left\{f: \mathbb{R}^{m \times n} \rightarrow \mathbb{R}:\left.f\right|_{E} \leq 0\right\} .
\end{aligned}
$$

We then have respectively, the convex, polyconvex, rank one convex, rank one convex finite and (closure of the) quasiconvex hull defined by

$$
\begin{aligned}
\operatorname{co} E & =\left\{\xi \in \mathbb{R}^{m \times n}: f(\xi) \leq 0, \text { for every convex } f \in \overline{\mathcal{F}}_{E}\right\} \\
\text { Pco } E & =\left\{\xi \in \mathbb{R}^{m \times n}: f(\xi) \leq 0, \text { for every polyconvex } f \in \overline{\mathcal{F}}_{E}\right\} \\
\operatorname{Rco} E & =\left\{\xi \in \mathbb{R}^{m \times n}: f(\xi) \leq 0, \text { for every rank one convex } f \in \overline{\mathcal{F}}_{E}\right\} \\
\operatorname{Rco}_{f} E & =\left\{\xi \in \mathbb{R}^{m \times n}: f(\xi) \leq 0, \text { for every rank one convex } f \in \mathcal{F}_{E}\right\} \\
\overline{\mathrm{Qco}} E & =\left\{\xi \in \mathbb{R}^{m \times n}: f(\xi) \leq 0, \text { for every quasiconvex } f \in \mathcal{F}_{E}\right\} .
\end{aligned}
$$

Remark 6 The definition of rank one convex hull Rco $E$ that we adopted is called, by some authors, lamination convex hull of $E$; while the same authors call $\mathrm{Rco}_{f} \mathrm{E}$ the rank one convex hull of $E$.

We start by pointing out several important facts.

1) The definition of convex hull is equivalent to the classical one, i.e. the smallest convex set that contains $E$. In fact it is enough to consider only one function: the convex envelope, $C \chi_{E}\left(=\chi_{\operatorname{co} E}\right)$, of the indicator function of the set $E$, namely

$$
\chi_{E}(\xi)=\left\{\begin{array}{cc}
0 & \text { if } \xi \in E \\
+\infty & \text { if } \xi \notin E .
\end{array}\right.
$$

2) If we replace $\overline{\mathcal{F}}_{E}$ by $\mathcal{F}_{E}$ in the definition of co $E$, we get $\overline{\operatorname{co}} E$ the closure of the convex hull.

3) Similar considerations apply to the polyconvex hull, in particular it is sufficient to consider the polyconvex envelope, $P \chi_{E}$, of the indicator function of the set $E$.

4) If the set $E$ is compact then so are co $E$ and Pco $E$.

5) We also have as a consequence of Carathéodory theorem

Proposition 7 Let $E \subset \mathbb{R}^{m \times n}$, then the following representation hold

$$
\begin{gathered}
\operatorname{co} E=\left\{\xi \in \mathbb{R}^{m \times n}: \xi=\sum_{i=1}^{m n+1} t_{i} \xi_{i}, \xi_{i} \in E, t_{i} \geq 0 \text { with } \sum_{i=1}^{m n+1} t_{i}=1\right\} \\
\operatorname{Pco} E=\left\{\xi \in \mathbb{R}^{m \times n}: T(\xi)=\sum_{i=1}^{\tau+1} t_{i} T\left(\xi_{i}\right), \xi_{i} \in E, t_{i} \geq 0 \text { with } \sum_{i=1}^{\tau+1} t_{i}=1\right\} .
\end{gathered}
$$


6) Matters are however very different with the other definitions, but let us first start with some resemblances.

Proposition 8 Let $E \subset \mathbb{R}^{m \times n}$ and set $R_{0} \operatorname{co} E=E$, and let for $i \in \mathbb{N}$

$$
\begin{aligned}
R_{i+1} \operatorname{co} E & =\left\{\xi \in \mathbb{R}^{m \times n}: \xi=t \xi_{1}+(1-t) \xi_{2},\right. \\
\xi_{1}, \xi_{2} & \left.\in R_{i} \operatorname{co} E, \operatorname{rank}\left\{\xi_{1}-\xi_{2}\right\}=1, t \in[0,1]\right\} .
\end{aligned}
$$

Then

$$
\operatorname{Rco} E=\cup_{i \in \mathbb{N}} R_{i} \operatorname{co} E .
$$

7) The rank one convex hull is equivalently defined through the rank one convex envelope, $R \chi_{E}$, of the indicator function of the set $E$ and it is the smallest rank one convex set that contains $E$.

We now turn our attention to some differences of behaviour among these notions.

8) Contrary to co $E$ and Pco $E$, if the set $E$ is compact then Rco $E$ is not necessarily compact as was pointed out by Kolar (18).

9) Contrary to $\operatorname{co} E$ and Pco $E$, the set Rco $f E$ is, in general (see below for an example), strictly larger than the closure of Rco $E$, i.e.,

$$
\overline{\mathrm{Rco}} E \varsubsetneqq \mathrm{Rco}_{f} E .
$$

10) There is no good definition of the quasiconvex hull if we replace $\mathcal{F}_{E}$ by $\overline{\mathcal{F}}_{E}$, since quasiconvex functions with values in $\overline{\mathbb{R}}=\mathbb{R} \cup\{+\infty\}$ are not yet well understood. In particular if $E=\left\{\xi_{1}, \xi_{2}\right\} \subset \mathbb{R}^{m \times n}$, then $Q \chi_{E}=\chi_{E}$, independently of the fact that $\xi_{1}-\xi_{2}$ is of rank one or not.

11) For any set $E \subset \mathbb{R}^{m \times n}$ we have

$$
\begin{gathered}
E \subset \operatorname{Rco} E \subset \mathrm{P} \operatorname{Po} E \subset \operatorname{co} E \\
\bar{E} \subset \overline{\mathrm{Rco}} E \subset \mathrm{Rco}_{f} E \subset \overline{\mathrm{Q} c o} E \subset \overline{\mathrm{P} c o} E \subset \overline{\operatorname{co}} E .
\end{gathered}
$$

We now discuss two examples that might shed some light on some differences between these new hulls and the convex one. In both examples we will consider the case $m=n=2$ and denote by $\mathbb{R}_{d}^{2 \times 2}$ the set of $2 \times 2$ diagonal matrices, we will write any such matrix as a vector of $\mathbb{R}^{2}$.

Example 9 The first example is by now classical and is due to Tartar and a very similar one by Casadio (cf. Example 2 page 116 in (5)). It shows the difference between $\operatorname{Rco}_{f} E$ and Rco $E$. Let $E=\left\{\xi_{1}, \xi_{2}, \xi_{3}, \xi_{4}\right\} \subset \mathbb{R}_{d}^{2 \times 2}$ be defined by

$$
\xi_{1}=(2,1), \xi_{2}=(1,-2), \xi_{3}=(-2,-1), \xi_{4}=(-1,2) .
$$


It is easy to see that since $\operatorname{rank}\left\{\xi_{i}-\xi_{j}\right\}=2$ for every $i \neq j$, then $E=\operatorname{Rco} E$. However

$$
\begin{aligned}
\operatorname{Rco}_{f} E & =\left\{\xi \in \mathbb{R}_{d}^{2 \times 2}: \xi=(x, y) \in[-1,1]^{2}\right\} \\
& \cup\left\{\xi \in \mathbb{R}_{d}^{2 \times 2}: \xi=(x, 1), x \in[1,2]\right\} \\
& \cup\left\{\xi \in \mathbb{R}_{d}^{2 \times 2}: \xi=(1, y), y \in[-2,-1]\right\} \\
& \cup\left\{\xi \in \mathbb{R}_{d}^{2 \times 2}: \xi=(x,-1), x \in[-2,-1]\right\} \\
& \cup\left\{\xi \in \mathbb{R}_{d}^{2 \times 2}: \xi=(-1, y), y \in[1,2]\right\} .
\end{aligned}
$$

The second example exhibits another peculiarity of the rank one convex hull that is very different from the convex one. It is also a phenomenon that one wants to avoid when applying the results to the analytical problem discussed in the Introduction.

Example 10 Let $E=\left\{\xi_{1}, \xi_{2}, \xi_{3}, \xi_{4}, \xi_{5}, \xi_{6}\right\} \subset \mathbb{R}_{d}^{2 \times 2}$ be defined by

$\xi_{1}=(1,0), \xi_{2}=(1,-1), \xi_{3}=(0,-1), \xi_{4}=(-1,0), \xi_{5}=(-1,1), \xi_{6}=(0,1)$.

It is easy to find that

$$
\operatorname{Rco} E=\{\xi: \xi=(x, y) \in[0,1] \times[-1,0]\} \cup\{\xi: \xi=(x, y) \in[-1,0] \times[0,1]\}
$$

and its interior (relative to $\mathbb{R}_{d}^{2 \times 2}$ ) is given by

$\operatorname{int} \operatorname{Rco} E=\{\xi: \xi=(x, y) \in(0,1) \times(-1,0)\} \cup\{\xi: \xi=(x, y) \in(-1,0) \times(0,1)\}$.

However there is no way of finding a set $E_{\delta}$ with the following "approximation property" (cf. (9) and (10) for more details concerning the use of this property):

(1) $E_{\delta} \subset \operatorname{Rco} E_{\delta} \subset \operatorname{int} \operatorname{Rco} E$ for every $\delta>0$;

(2) for every $\epsilon>0$ there exists $\delta_{0}=\delta_{0}(\epsilon)>0$ such that $\operatorname{dist}(\eta ; E) \leq \epsilon$ for every $\eta \in E_{\delta}$ and $\delta \in\left[0, \delta_{0}\right]$;

(3) if $\eta \in \operatorname{int} \operatorname{Rco} E$ then $\eta \in$ Rco $E_{\delta}$ for every $\delta>0$ sufficiently small.

In fact $\mathrm{Rco} E_{\delta}$ will be reduced, at best, to four segments and will have empty interior (and condition (3) will be violated).

\section{The singular values}

One of the most general example of such hulls concern sets that involve singular values. Let us first recall that the singular values of a given matrix $\xi \in \mathbb{R}^{n \times n}$, denoted by $0 \leq \lambda_{1}(\xi) \leq \ldots \leq \lambda_{n}(\xi)$, are the eigenvalues of $\left(\xi \xi^{t}\right)^{1 / 2}$.

We will consider three types of sets, letting $0<\gamma_{1} \leq \ldots \leq \gamma_{n}$ and $\alpha \leq \beta$ with $\alpha \neq 0$,

$$
\begin{gathered}
E=\left\{\xi \in \mathbb{R}^{n \times n}: \lambda_{i}(\xi)=\gamma_{i}, i=1, \ldots, n\right\} \\
E_{\alpha}=\left\{\xi \in \mathbb{R}^{n \times n}: \lambda_{i}(\xi)=\gamma_{i}, i=1, \ldots, n, \operatorname{det} \xi=\alpha\right\}
\end{gathered}
$$




$$
E_{\alpha, \beta}=\left\{\xi \in \mathbb{R}^{n \times n}: \lambda_{i}(\xi)=\gamma_{i}, i=2, \ldots, n, \operatorname{det} \xi \in\{\alpha, \beta\}\right\}
$$

where, since $|\operatorname{det} \xi|=\prod_{i=1}^{n} \lambda_{i}(\xi)$ and the singular values are ordered as $0 \leq$ $\lambda_{1}(\xi) \leq \ldots \leq \lambda_{n}(\xi)$, we should respectively impose in the second and third cases that

$$
\begin{gathered}
\prod_{i=1}^{n} \gamma_{i}=|\alpha| \\
\gamma_{2} \prod_{i=2}^{n} \gamma_{i} \geq \max \{|\alpha|,|\beta|\} .
\end{gathered}
$$

Note that the third case contains the other ones as particular cases. Indeed the first one is deduced from the last one by setting $\beta=-\alpha$ and

$$
\gamma_{1}=\beta\left[\prod_{i=2}^{n} \gamma_{i}\right]^{-1}
$$

while the second one is obtained by setting $\beta=\alpha$ and

$$
\gamma_{1}=|\alpha|\left[\prod_{i=2}^{n} \gamma_{i}\right]^{-1}
$$

in the third case.

Our result (cf. (9), (12) for the two first ones and for the third case: Dacorogna-Ribeiro (11)) is then

Theorem 11 Under the above conditions and notations the following set of identities holds

$$
\begin{gathered}
\operatorname{co} E=\left\{\xi \in \mathbb{R}^{n \times n}: \sum_{i=\nu}^{n} \lambda_{i}(\xi) \leq \sum_{i=\nu}^{n} \gamma_{i}, \nu=1, \ldots, n\right\} \\
\text { Pco } E=\overline{\operatorname{Qco}} E=\operatorname{Rco} E=\left\{\xi \in \mathbb{R}^{n \times n}: \prod_{i=\nu}^{n} \lambda_{i}(\xi) \leq \prod_{i=\nu}^{n} \gamma_{i}, \nu=1, \ldots, n\right\} . \\
\text { Pco } E_{\alpha}=\operatorname{Rco} E_{\alpha}=\left\{\xi \in \mathbb{R}^{n \times n}: \prod_{i=\nu}^{n} \lambda_{i}(\xi) \leq \prod_{i=\nu}^{n} \gamma_{i}, \nu=2, \ldots, n, \operatorname{det} \xi=\alpha\right\} . \\
\text { Pco } E_{\alpha, \beta}=\operatorname{Rco} E_{\alpha, \beta}=\left\{\xi \in \mathbb{R}^{n \times n}: \prod_{i=\nu}^{n} \lambda_{i}(\xi) \leq \prod_{i=\nu}^{n} \gamma_{i}, \nu=2, \ldots, n, \operatorname{det} \xi \in[\alpha, \beta]\right\} .
\end{gathered}
$$

As it was pointed out by Buliga (2) there is a surprising formal connection, still not well understood, between the above theorem (with $\alpha=\beta$ ) and some classical results of H. Weyl, A. Horn and C.J. Thompson (see (15), (16) page 171 or (19)). The result states that if we denote, as above, the singular values of a given matrix $\xi \in \mathbb{R}^{n \times n}$ by $0 \leq \lambda_{1}(\xi) \leq \ldots \leq \lambda_{n}(\xi)$ and its eigenvalues, 
which are complex in general, by $\mu_{1}(\xi), \ldots, \mu_{n}(\xi)$ and if we order them by their modulus $\left(0 \leq\left|\mu_{1}(\xi)\right| \leq \ldots \leq\left|\mu_{n}(\xi)\right|\right)$ then the following result holds

$$
\begin{aligned}
& \prod_{i=\nu}^{n}\left|\mu_{i}(\xi)\right| \leq \prod_{i=\nu}^{n} \lambda_{i}(\xi), \nu=2, \ldots, n \\
& \prod_{i=1}^{n}\left|\mu_{i}(\xi)\right|=\prod_{i=1}^{n} \lambda_{i}(\xi)
\end{aligned}
$$

for any matrix $\xi \in \mathbb{R}^{n \times n}$.

Acknowledgements. We have benefitted from the financial support of the Fonds National Suisse (grant 21-61390.00)

\section{References}

[1] Bressan A. and Flores F., On total differential inclusions; Rend. Sem. Mat. Univ. Padova, 92 (1994), 9-16.

[2] Buliga M., Majorisation with applications in elasticity; to appear.

[3] Cellina A., On minima of a functional of the gradient: sufficient conditions, Nonlinear Anal. Theory Methods Appl., 20 (1993), 343-347.

[4] Croce G., A differential inclusion: the case of an isotropic set, to appear.

[5] Dacorogna B., Direct methods in the calculus of variations, Applied Math. Sciences, 78, Springer, Berlin (1989).

[6] Dacorogna B. and Marcellini P., Théorèmes d'existence dans le cas scalaire et vectoriel pour les équations de Hamilton-Jacobi, C.R. Acad. Sci. Paris, 322 (1996), 237-240.

[7] Dacorogna B. and Marcellini P., General existence theorems for HamiltonJacobi equations in the scalar and vectorial case, Acta Mathematica, 178 (1997), 1-37.

[8] Dacorogna B. and Marcellini P., On the solvability of implicit nonlinear systems in the vectorial case; in the AMS Series of Contemporary Mathematics, edited by G.Q. Chen and E. DiBenedetto, (1999), 89-113.

[9] Dacorogna B. and Marcellini P., "Implicit partial differential equations"; Birkhäuser, Boston, (1999).

[10] Dacorogna B. and Pisante G., A general existence theorem for differential inclusions in the vector valued case; to appear in Abstract and Applied Analysis.

[11] Dacorogna B. and Ribeiro A.M., Existence of solutions for some implicit pdes and applications to variational integrals involving quasiaffine functions; to appear. 
[12] Dacorogna B. and Tanteri C., Implicit partial differential equations and the constraints of nonlinear elasticity; J. Math. Pures Appl. 81 (2002), 311-341.

[13] De Blasi F.S. and Pianigiani G., On the Dirichlet problem for HamiltonJacobi equations. A Baire category approach; Nonlinear Differential Equations Appl. 6 (1999), 13-34.

[14] Friesecke G., A necessary and sufficient condition for non attainment and formation of microstructure almost everywhere in scalar variational problems, Proc. Royal Soc. Edinburgh, 124A (1994), 437-471.

[15] Horn R.A. and Johnson C.A.: Matrix Analysis; Cambridge University Press (1985).

[16] Horn R.A. and Johnson C.A.: Topics in Matrix Analysis; Cambridge University Press (1991).

[17] Kirchheim B., Deformations with finitely many gradients and stability of quasiconvex hulls; C. R. Acad. Sci. Paris, 332 (2001), 289-294.

[18] Kolar J., Non compact lamination convex hulls; Ann. I. H. Poincaré, AN 20 (2003), 391-403.

[19] Marshall A. W. and Olkin I., Inequalities: theory of majorisation and its applications; Academic Press (1979).

[20] Müller S. and Sverak V., Attainment results for the two-well problem by convex integration; ed. Jost J., International Press, (1996), 239-251.

[21] Müller S. and Sychev M., Optimal existence theorems for nonhomogeneous differential inclusions; J. Funct. Anal. 181 (2001), 447-475.

[22] Sychev M., Comparing two methods of resolving homogeneous differential inclusions; Calc. Var. Partial Differential Equations 13 (2001), 213-229. 